\title{
Development of an Advanced Fuzzy Active Steering Controller and a Novel Method to Tune the Fuzzy Controller
}

\author{
Kiumars Jalali, Thomas Uchida, John McPhee and Steve Lambert \\ Univ. of Waterloo
}

\begin{abstract}
A two-passenger, all-wheel-drive urban electric vehicle (AUTO21EV) with four direct-drive in-wheel motors has been designed and developed at the University of Waterloo. An advanced genetic-fuzzy active steering controller is developed based on this vehicle platform. The rule base of the fuzzy controller is developed from expert knowledge, and a multicriteria genetic algorithm is used to optimize the parameters of the fuzzy active steering controller. To evaluate the performance of this controller, a computational model of the AUTO21EV is driven through several standard test maneuvers using an advanced path-following driver model. As the final step in the evaluation process, the genetic-fuzzy active steering controller is implemented in a hardware- and operator-in-the-loop driving simulator to confirm its performance and effectiveness.
\end{abstract}

CITATION: Jalali, K., Uchida, T., McPhee, J. and Lambert, S., "Development of an Advanced Fuzzy Active Steering Controller and a Novel Method to Tune the Fuzzy Controller," SAE Int. J. Passeng. Cars - Electron. Electr. Syst. 6(1):2013, doi:10.4271/2013-01-0688.

\section{INTRODUCTION}

Recently, electric vehicles (EVs) have attracted a great deal of interest as an elegant solution to environmental and energy concerns. Among other benefits, EVs are more efficient and can reduce or eliminate the environmental noise and pollutants that are associated with conventional internal combustion engine (ICE) vehicles considerably. Also, thanks to substantial improvements in electric motor and battery technologies, EVs now have driving performance metrics that are comparable to those of ICE vehicles. In addition, EVs are more appealing platforms on which to apply advanced motion control techniques, since the motor speed and torque can be generated and controlled almost instantly, and more accurately than any ICE or even hydraulic brake system.

Figure 1 illustrates the AUTO21EV, which is a twopassenger, all-wheel-drive urban electric vehicle that has been developed and modeled in this work using the ADAMS/ View and MapleSim software packages. This vehicle has a configuration similar to that of the commercially-available Smart fortwo, but is equipped with four direct-drive in-wheel motors and an active steering system on the front axle. Table 1 lists some of the relevant parameters used in the AUTO21EV model. The use of small but powerful directdrive in-wheel motors allows for the implementation of the most advanced all-wheel-drive system in which the optimal traction force can be generated on all tires by controlling the tire slips at all times.

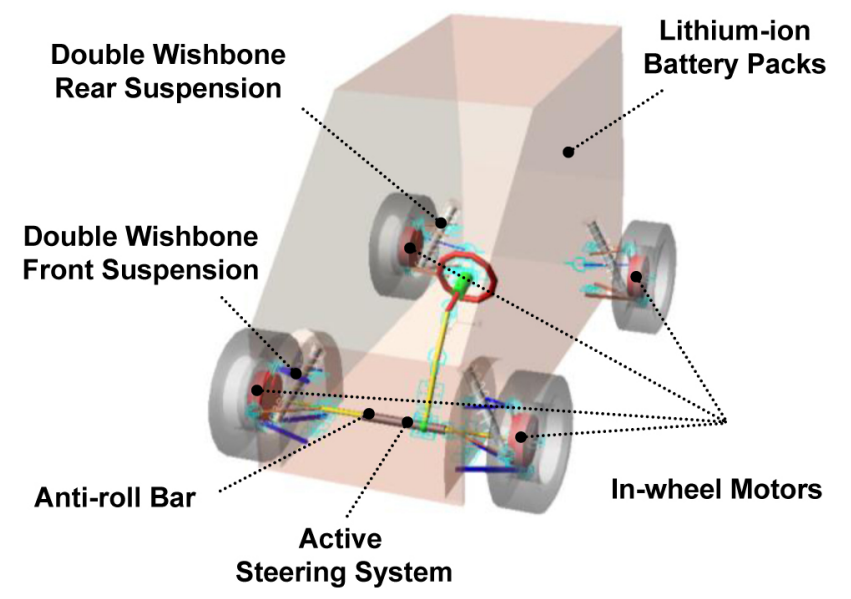

Figure 1. AUTO21EV concept vehicle

In 2004, BMW introduced its first commercial active steering system in its 5 -series class of vehicles [1]. Active steering bridges the gap between conventional steering systems and steer-by-wire technologies. Although an active steering system allows for driver-independent steering intervention, the mechanical linkage between the steering wheel and the rack-and-pinion system on the front axle 
remains in place, acting as a fail-safe mechanism. An active steering system facilitates two major functions: realizing a variable steering ratio, and maintaining vehicle stability and maneuverability during emergency maneuvers or when driving conditions call for a change in steering response.

Table 1. AUTO21EV model parameters

\begin{tabular}{|l|c|c|}
\hline Parameter & Value & Unit \\
\hline Vehicle mass $(M)$ & 1075 & $\mathrm{~kg}$ \\
\hline Vehicle yaw moment of inertia $\left(\mathrm{I}_{\mathrm{zz}}\right)$ & 1171 & $\mathrm{~kg} \mathrm{~m}$ \\
\hline Distance from CG to front axle $(\mathrm{a})$ & 0.82 & $\mathrm{~m}$ \\
\hline Distance from CG to rear axle $(\mathrm{b})$ & 0.98 & $\mathrm{~m}$ \\
\hline Front track width $\left(\mathrm{t}_{\mathrm{f}}\right)$ & 1.275 & $\mathrm{~m}$ \\
\hline Rear track width $\left(\mathrm{t}_{\mathrm{r}}\right)$ & 1.35 & $\mathrm{~m}$ \\
\hline Front tire cornering stiffness $\left(\mathrm{C}_{\alpha \mathrm{F}}\right)$ & 45570 & $\mathrm{~N} / \mathrm{rad}$ \\
\hline Rear tire cornering stiffness $\left(\mathrm{C}_{\alpha \mathrm{R}}\right)$ & 45570 & $\mathrm{~N} / \mathrm{rad}$ \\
\hline Tire size & $175 / 55 \mathrm{R} 15$ & - \\
\hline Tire unloaded radius $(\mathrm{r})$ & 0.29 & $\mathrm{~m}$ \\
\hline
\end{tabular}

The fundamental design conflict faced by conventional steering systems involves choosing a suitable geometric steering ratio, which affects not only the steering effort in the maneuvering range, but also the vehicle dynamics at higher speeds. An active steering system resolves this conflict by increasing the steering ratio at higher speeds to enhance the responsiveness of the vehicle, while decreasing it at lower speeds to enhance the maneuverability. The variable steering ratio is realized using a double planetary gear and an electric motor, adding additional steering angle to the driver's input at low speeds and counter-steering slightly at higher speeds. This property not only avoids hand-over-hand steering when parking, but also ensures that an essentially constant steering effort is required in the medium- and high-speed ranges.

Another substantial advantage of an active steering system is its ability to electronically augment the driver's steering input to stabilize the vehicle. In general, steering intervention can provide a faster response than braking individual wheels, as is done by an electronic stability control (ESC) system, since it takes time to build up hydraulic brake pressure. In contrast to braking intervention, controlling the vehicle using steering intervention is generally considered to be a continuous process and is not perceived by the driver-or, at least, is not perceived as being disruptive or annoying [1]. Figure 2 compares the effectiveness of an ESC system to that of an active steering system for correcting an oversteered vehicle, where the rear tires have reached their limit of adhesion during a left turn. In such a situation, an ESC system applies a braking force $\left(\mathrm{F}_{\mathrm{B}}\right)$ to the front-outside tire to generate the required corrective yaw moment, which is calculated as follows [2]]:

$$
M_{z, B}=F_{B} \cdot \frac{t_{f}}{2}
$$

where $t_{f}$ is the front track width of the vehicle. An active steering system, on the other hand, applies a counter-steering angle in order to generate the same corrective yaw moment, but does so using the lateral forces of the front tires:

$$
M_{z, A S} \approx 2 F_{Y} \cdot a
$$

where $\mathrm{a}$ is the distance between the front axle and the vehicle center of mass. Assuming that the same corrective yaw moment is generated by these two systems, and knowing that the front track width $\left(t_{f}\right)$ of most passenger cars is approximately equal to the distance between the center of gravity and the front axle (a), the required lateral force $\left(\mathrm{F}_{\mathrm{Y}}\right)$ on the front tires is only one-fourth of the required braking force $\left(\mathrm{F}_{\mathrm{B}}\right)$ :

$$
M_{z, B}=M_{z, A S} \Rightarrow F_{Y} \approx \frac{F_{B}}{4}
$$

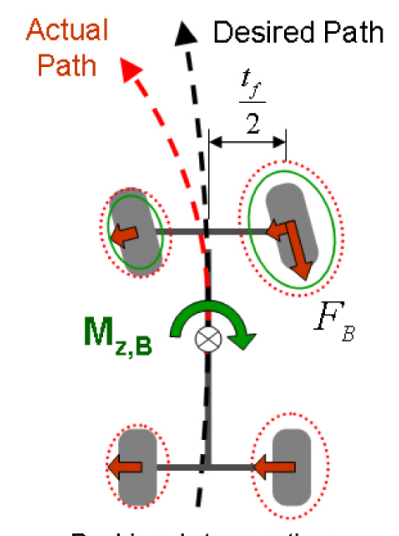

Braking Intervention

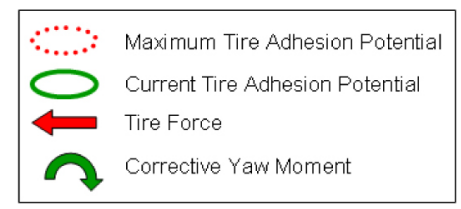

Figure 2. Generation of a corrective yaw moment by an ESC system through braking intervention (left) and by an active steering system through steering angle intervention (right)

Therefore, it is advantageous to use steering intervention rather than braking intervention to generate a corrective yaw moment when controlling a vehicle on slippery surfaces, where the limits of adhesion can be easily reached. Active steering is also effective at correcting the side-pushing effect that occurs when driving on a $\mu$-split road (due to the different traction forces on the two sides of a vehicle); however, an active steering system is only effective in a limited range due to the limitations of the actuators. For instance, BMW's active steering system is only able to apply up to 3 degrees of steering angle on the front wheels, which is 
equivalent to a driver steering wheel input of about 55 degrees [1].

Almost every active steering system on the market today is based on the classical proportional-integral-derivative (PID) control system $[\underline{1}, \underline{2}, \underline{3}, \underline{4}, \underline{5}, \underline{6}]$. In general, tuning the gains of such a PID controller requires extensive and rigorous field tests that are conducted by vehicle experts in a car manufacturing company. In this work, however, we develop a novel fuzzy active steering controller and a reliable method to tune its membership functions in an optimized way, which makes expensive field testing unnecessary for the most part.

\section{DEVELOPMENT OF AN ADVANCED FUZZY STEERING CONTROLLER}

A complete steering system has been developed for the AUTO21EV in the ADAMS/View environment by Bode [7], where the kinematics and dynamics of the steering system have been analyzed. This steering system has a 55\% Ackermann behavior in order to allow for a smaller turning radius and a higher lateral force capacity on the front-outside tire when moving laterally at higher speeds. The nonlinear characteristics of the steering system have been implemented in a MapleSim model of the AUTO21EV using a look-up table and an independent motion driver for each wheel on the front axle.

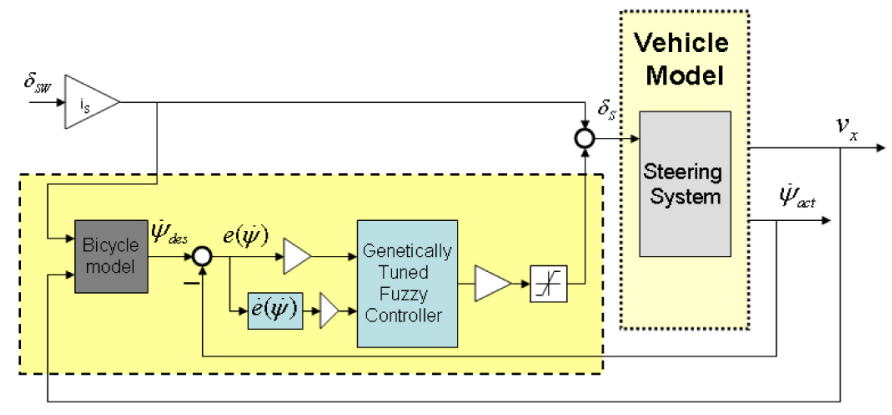

Figure 3. Block diagram of the fuzzy active steering system developed for the AUTO21EV

Fuzzy logic control systems are robust and flexible inference methods that are effective at tackling complicated nonlinear dynamic control problems. As such, they are ideally suited for controlling the highly nonlinear behavior inherent in vehicular dynamics. Fuzzy control systems can tolerate imprecise information and can describe expert knowledge in vague linguistic terms, which suits the subjective nature of vehicle control design [ 8$]$ ] and active steering systems in particular. With this in mind, a fuzzy active steering system is developed based on the AUTO21EV steering system. A multi-criteria genetic algorithm is used to optimize the shapes and distributions of the fuzzy membership functions associated with the input and output variables in order to minimize the vehicle trajectory, yaw rate, and sideslip angle errors. The rule base of the fuzzy active steering system is described in vague linguistic terms using expert knowledge, which suits the subjective nature of vehicle stability and handling. Shown in Figure 3 is a block diagram of the fuzzy active steering system. The inputs to the fuzzy controller are the yaw rate error $\mathrm{e}(\dot{\psi})$ and the rate of change of the yaw rate error $\dot{\mathrm{e}}(\dot{\psi})$; the output of the controller is the steering angle required to correct the driver's steering input.

Many researchers have claimed that information about the yaw rate alone is not always sufficient to identify an unstable vehicle operating at its physical limit, and argue that more comprehensive control can be achieved by simultaneously considering the vehicle sideslip angle, to which the driver is particularly sensitive (preferring small angles [9]). Since the active steering system has a limited range of effectiveness, however, knowledge of the sideslip angle is not expected to be of substantial benefit in emergency situations. In other words, in cases where a high sideslip angle is likely, an active steering system would not be stabilizing the vehicle on its own. Instead, an active steering system would act as a complementary system, helping to stabilize the vehicle in collaboration with other active chassis subsystems $[\underline{10}, 11]$. With this in mind, a linear bicycle model is used as a reference model to calculate the desired yaw rate of the vehicle, to which the controller attempts to match the nonlinear behavior of the vehicle. The desired yaw rate is computed as a function of the front axle steering angle $\left(\delta_{\mathrm{w}}\right)$ and the vehicle forward velocity $\left(\mathrm{v}_{\mathrm{x}}\right)$ as follows:

$$
\dot{\psi}_{\text {des }}=\frac{v_{x}}{\left(L+K_{u s} \times v_{x}^{2}\right)} \times \tan \left(\delta_{w}\right)
$$

The understeering gradient of the vehicle $\left(\mathrm{K}_{\mathrm{us}}\right)$ is calculated as follows:

$$
K_{u s}=\frac{M}{L} \times\left(\frac{b}{C_{a F}}-\frac{a}{C_{a R}}\right)
$$

where $\mathrm{L}$ is the wheelbase, $\mathrm{M}$ is the curb mass of the vehicle, a and $b$ are the distances of the front and rear axles from the vehicle center of mass, and $\mathrm{C}_{\alpha \mathrm{F}}$ and $\mathrm{C}_{\alpha \mathrm{R}}$ are the lateral stiffnesses of the front and rear tires, respectively. Using equation (5), the understeering gradient of the AUTO21EV is determined to be $0.0021 \mathrm{rad} / \mathrm{m} / \mathrm{s}^{2}$. Since the lateral acceleration of the vehicle is fundamentally limited by the friction coefficient between the tires and the road, the maximum desired yaw rate is also limited. The steady-state lateral acceleration of the vehicle can be expressed as follows [12]:

$$
\left|a_{y}\right|=\left|\frac{v_{x}^{2}}{R}\right|=\left|\dot{\psi}_{\text {des }} \times v_{x}\right| \leq\left(\mu_{y, \max } \times g\right)
$$

where $\mathrm{R}$ is the radius of curvature, $\mathrm{g}$ is the acceleration due to gravity, and $\mu_{y, \max }$ is the maximum lateral friction coefficient 
between the tire and the road. The desired yaw rate must, therefore, be limited by the following value:

$$
\left|\dot{\psi}_{d e s}\right| \leq\left|\frac{\mu_{y \text { max }} \times g}{v_{x}}\right|
$$

It is important to note that the actual yaw rate of the vehicle can be measured directly using a gyroscope.

The definitions of the input and output variables used in the fuzzy active steering system are provided in Table 2. In the fuzzification stage, the controller inputs are normalized to simplify the definition of the fuzzy sets. To provide sufficient rule coverage, five fuzzy sets are used for the yaw rate error and the rate of change of the yaw rate error. The output of the fuzzy controller must be defuzzified and scaled before being used by the steering system. Nine fuzzy sets are used to describe the output of the controller, which is the required corrective steering angle. A Mamdani fuzzy inference system [8] processes the inputs through the list of rules in the knowledge base and calculates the output using the following fuzzy rule pattern:

$$
\text { IF } \mathrm{e}(\dot{\psi}) \text { is } A \text { AND } \dot{\mathrm{e}}(\dot{\psi}) \text { is } B \text { THEN } \delta_{\text {corr }} \text { is } C
$$

where $A, B$, and $C$ are fuzzy sets defined on the input and output domains. Figure 4 illustrates the fuzzy rule base and the corresponding control surface for the fuzzy active steering system using the initial untuned fuzzy membership functions; the linguistic terms that have been used are listed in Table 3 . The initial shapes and distributions of the membership functions used for the input and output variables of the fuzzy controller are illustrated in Figure 5. The fuzzy rules developed for this controller are determined based on expert knowledge and extensive investigation into the behavior of the vehicle in different driving conditions. These fuzzy rules are formed using fuzzy variables whose membership functions are of unknown shapes, sizes, and relative positions. Since a fuzzy controller is unable to learn or adapt to its environment on its own (and instead of resorting to tuning the membership functions of the fuzzy controller manually, which is an inefficient, arduous, and timeconsuming task), a multi-criteria genetic algorithm is used in this work to find the ideal shapes and relative positions of the membership functions.

Table 2. Definition of the input and output variables of the fuzzy active steering controller

\begin{tabular}{|c|c|}
\hline Variable & Definition \\
\hline Input 1 & $e(\dot{\psi})=\dot{\psi}_{\text {desired }}-\dot{\psi}_{\text {actual }}$ \\
\hline Input 2 & $\dot{e}_{k}(\dot{\psi})=\left(e_{k}(\dot{\psi})-e_{k-1}(\dot{\psi})\right) / T_{\text {sampling }}$ \\
\hline Output & $\delta_{\text {corrective }}$ \\
\hline
\end{tabular}

Table 3. Linguistic variables used in the fuzzy rules

\begin{tabular}{|c|c|c|c|c|c|c|c|c|}
\hline \multirow{2}{*}{\multicolumn{2}{|c|}{$\begin{array}{l}\text { Control } \\
\text { Rule Base }\end{array}$}} & \multicolumn{7}{|c|}{$\mathrm{e}(\dot{\psi})$} \\
\hline & & NL & NM & NS & ZE & PS & PM & $\mathrm{PL}$ \\
\hline \multirow{7}{*}{$\frac{Ð}{2}$} & NL & NVL & NVL & $\mathrm{NL}$ & NM & NS & $\mathrm{ZE}$ & ZE \\
\hline & NM & NVL & NL & NM & NS & ZE & $\mathrm{ZE}$ & PS \\
\hline & NS & NL & $\mathrm{NM}$ & NS & ZE & ZE & PS & PM \\
\hline & ZE & NL & NM & NS & ZE & PS & $\mathrm{PM}$ & $\mathrm{PL}$ \\
\hline & PS & NM & NS & ZE & ZE & PS & PM & PL \\
\hline & PM & NS & ZE & ZE & PS & PM & PL & PVL \\
\hline & $\mathrm{PL}$ & ZE & ZE & PS & PM & $\mathrm{PL}$ & PVL & PVL \\
\hline
\end{tabular}

\begin{tabular}{|c|c|}
\hline Acronym & Linguistic Variable \\
\hline NVL & Negative Very Large \\
\hline NL & Negative Large \\
\hline NM & Negative Medium \\
\hline NS & Negative Small \\
\hline ZE & Zero \\
\hline PS & Positive Small \\
\hline PM & Positive Medium \\
\hline PL & Positive Large \\
\hline PVL & Positive Very Large \\
\hline
\end{tabular}

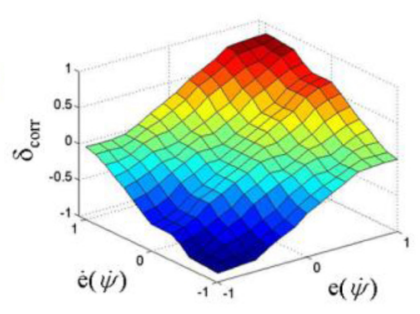

Figure 4. Control rule base (left) and untuned control surface (right) of the fuzzy active steering controller
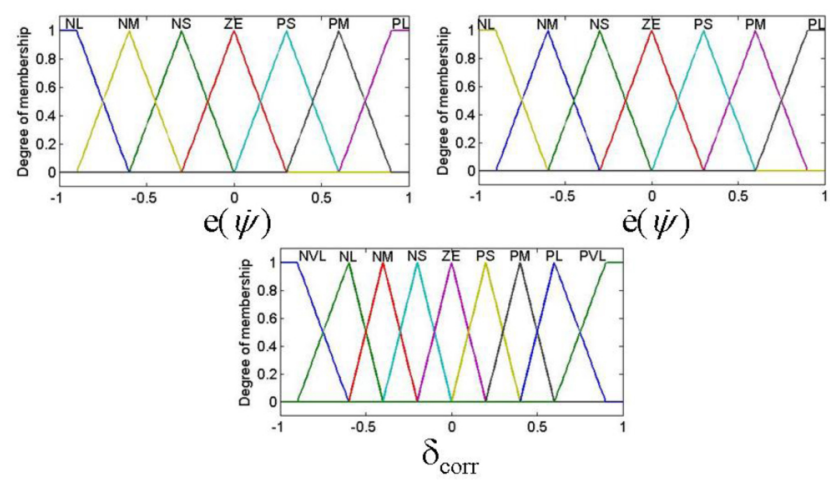

\section{Figure 5. Untuned shapes and distributions of the membership functions for the input and output variables of the fuzzy active steering controller}

A severe double-lane-change maneuver with obstacle avoidance was used to evaluate the effectiveness of each candidate controller. The specifications of a double-lanechange maneuver are described in the ISO 3888 specification [14] (Figure 6). In this test, the vehicle enters the course at a particular speed and the accelerator is released. The driver then attempts to negotiate the course without striking the cones. The test speed is progressively increased until either instability occurs or the course can no longer be negotiated successfully. Such a severe maneuver effectively demonstrates the cornering capability of a vehicle when driving at the friction limit in both directions and, therefore, many car manufacturers and research institutions consider this test to be a suitable maneuver for assessing ESC systems [13]. The ISO double-lane-change maneuver is typically performed as a closed-loop driving test, and is used to adjust 
the dynamics of a vehicle based on the subjective evaluations of professional drivers. Consequently, simulation of this maneuver demands a driver model [16] that can dynamically adjust the steering wheel according to the vehicle trajectory and yaw rate response at every time step of a simulation. Since the membership functions of the fuzzy controller must be tuned in a general sense and not based on the response of a specific driver or driver model, and also noting that the fuzzy controller is responsible only for the stability of the vehicle, the double-lane-change maneuver used in this work is performed as an open-loop driving test rather than a closedloop test.

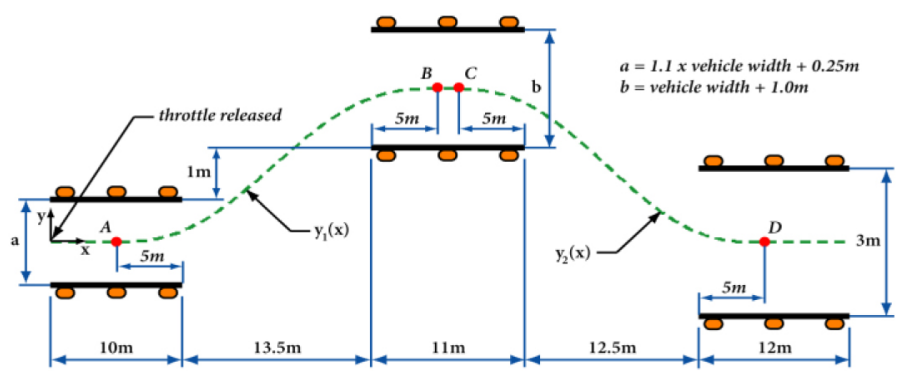

Figure 6. ISO 3888 double-lane-change maneuver test track [14]

Therefore, the double-lane-change maneuver test is defined by determining the desired trajectory and the corresponding steering input for a 'neutral-steer' vehicle driving through the ISO 3888 test track at a low speed. Such a steering input is considered to be the most desirable steering input; ideally, the driver should be able to successfully complete the double-lane-change maneuver with this input even at higher speeds. In this way, any deviation from the desired trajectory or desired yaw rate is considered to be a stability error that the fuzzy active steering controller should correct. The multi-criteria genetic algorithm is used to identify the ideal shapes and distributions of the membership functions for the fuzzy active steering controller such that the vehicle trajectory, yaw rate, and sideslip errors are minimized.

The desired vehicle trajectory, which is shown as a dashed line in Figure 6, is defined as a function of forward displacement $\mathrm{y}_{\mathrm{i}}(\mathrm{x})$ using two fifth-order splines that are connected with a straight line segment [7]:

$$
y(x)=\left\{\begin{array}{ccc}
0 & \text { if } & x<18.5 \\
\mathrm{c}_{1} \mathrm{x}^{5}+\cdots+\mathrm{c}_{5} \mathrm{x}+\mathrm{c}_{6} & \text { if } & 18.5 \leq x<35 \\
3.0425 & \text { if } & 35 \leq x<41.5 \\
\mathrm{c}_{7} \mathrm{x}^{5}+\cdots+\mathrm{c}_{11} \mathrm{x}+\mathrm{c}_{12} & \text { if } & 41.5 \leq x<61 \\
0 & \text { if } & x \geq 61
\end{array}\right.
$$

where $c_{1}$ to $c_{12}$ are the spline parameters, which are determined by applying boundary conditions to the splines. The steering input that can drive a 'neutral-steer' vehicle through this trajectory is defined as a function of the wheelbase $L$ and road curvature $\kappa(\mathrm{x})$ as follows:

$$
\delta(x)=L \times \kappa(x)=L \times \frac{\ddot{y}(x)}{\left(\dot{y}^{2}(x)+1\right)^{3 / 2}}
$$

where $\dot{y}(x)$ and $\ddot{y}(x)$ are the first and second derivatives of the vehicle trajectory $y(x)$, respectively. Figure 7 illustrates the required steering wheel angle for this maneuver. Note that the amplitude and frequency associated with the first lane change is larger, since the first turn is slightly tighter than the second. Figure 8 illustrates the desired and actual vehicle trajectories, yaw rates, and sideslip angles when driving through the double-lane-change maneuver with a fixed steering input, an initial speed of $25 \mathrm{~km} / \mathrm{h}$, and without an active steering controller. This figure demonstrates that the AUTO21EV is able to complete the maneuver at a low speed using the calculated fixed steering input.

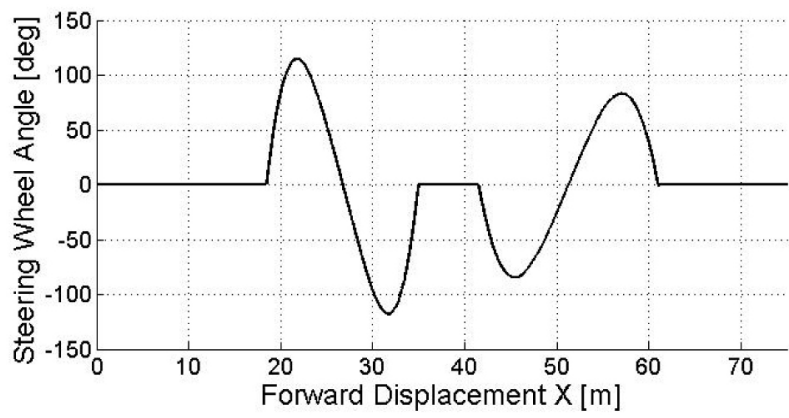

\section{Figure 7. Desired steering wheel input for driving through the double-lane-change maneuver when performed as an open-loop test}

The scaling function technique is chosen for the genetic tuning of the fuzzy membership functions. Scaling functions use a relatively small number of parameters to affect the shapes and overall distribution of the membership functions for a particular fuzzy variable. Hence, encoding these parameters results in shorter chromosomes and, consequently, shorter computation times. This method also guarantees that the adjacency constraint is met, which ensures that the sum of all membership functions is equal to unity for every point in the universe of discourse, and that a symmetrical distribution of the membership functions is maintained around the center of the universe of discourse. The following nonlinear scaling function is used for this application [15]:

$$
f(x)=\operatorname{sign}(x) \times|x|^{\alpha}
$$

where $\alpha$ is the scaling parameter, $\alpha>0$. This scaling function symmetrically increases $(\alpha>1)$ or decreases $(\alpha<1)$ the relative density of the membership functions around the origin, and has the opposite effect at the boundaries of the universe of discourse. Using this nonlinear scaling function 
and noting that the untuned membership functions are distributed symmetrically around the origin (Figure 5), the final tuned membership functions are guaranteed to be distributed symmetrically as well. Altogether, three scaling parameters are encoded for the input and output variables of the fuzzy active steering system. These encoded parameters are concatenated to generate a chromosome for the genetic tuning process.
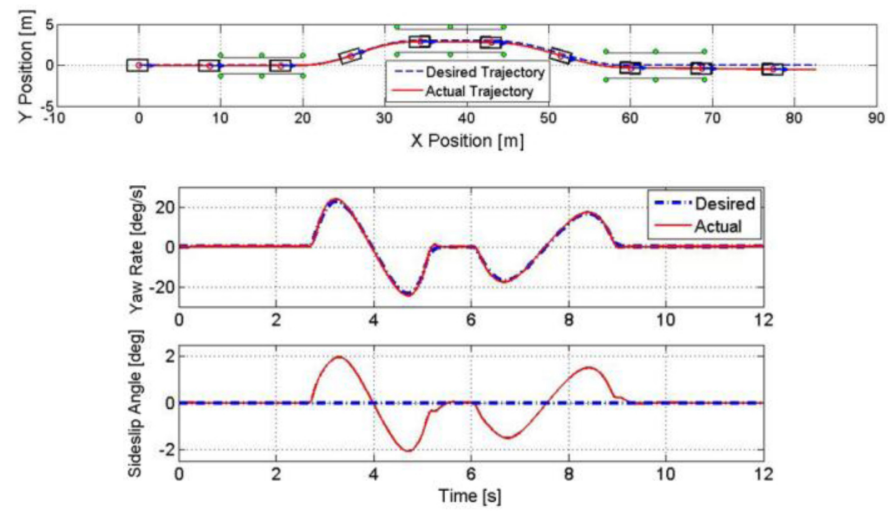

Figure 8. Desired and actual vehicle trajectories (top), yaw rates (center), and sideslip angles (bottom) of the AUTO21EV when driving through the double-lanechange maneuver with a fixed steering input, an initial speed of $25 \mathrm{~km} / \mathrm{h}$, and without an active steering controller

A genetic algorithm is particularly suitable for solving the multi-criteria optimization problem of tuning the input and output variables of the fuzzy active steering system, the objective of which is to minimize the mean-squared errors of the vehicle trajectory, yaw rate, and sideslip angle:

$$
\operatorname{MSE}(\text { trajectory })=\frac{1}{\mathrm{~N}} \sum_{\mathrm{k}=1}^{\mathrm{N}}\left[\mathrm{y}_{\text {desired }}\left(\mathrm{x}_{\mathrm{k}}\right)-\mathrm{y}_{\text {actual }}\left(\mathrm{x}_{\mathrm{k}}\right)\right]^{2}
$$

$$
\begin{aligned}
& \operatorname{MSE}(\text { yaw rate })=\frac{1}{\mathrm{~N}} \sum_{\mathrm{k}=1}^{\mathrm{N}}\left[\dot{\psi}_{\text {desired }}(\mathrm{k})-\dot{\psi}_{\text {actual }}(\mathrm{k})\right]^{2} \\
& \operatorname{MSE}(\text { sideslip })=\frac{1}{\mathrm{~N}} \sum_{\mathrm{k}=1}^{\mathrm{N}}\left[\beta_{\text {desired }}(\mathrm{k})-\beta_{\text {actual }}(\mathrm{k})\right]^{2}
\end{aligned}
$$

where $\mathrm{N}$ is the number of sample points, $\mathrm{y}_{\text {desired }}\left(\mathrm{x}_{\mathrm{k}}\right)$ and $\mathrm{y}_{\text {actual }}\left(\mathrm{x}_{\mathrm{k}}\right)$ are the desired and actual lateral positions of the vehicle for a given forward position $\mathrm{x}_{\mathrm{k}}, \dot{\psi}_{\text {desired }}(\mathrm{k})$ and $\dot{\psi}_{\text {actual }}(\mathrm{k})$ are the desired and actual vehicle yaw rates, and $\beta_{\text {desired }}(\mathrm{k})$ and $\beta_{\text {actual }}(\mathrm{k})$ are the desired and actual vehicle sideslip angles at a given time step, respectively. Since the objective of the genetic algorithm is to minimize all the aforementioned errors, the fitness function associated with each chromosome is defined as the weighted sum of the inverse of the resulting vehicle trajectory, yaw rate, and sideslip mean-squared errors:

$$
\text { Fitness }=\frac{\mathrm{w}_{1}}{\mathrm{MSE}(\text { trajectory })}+\frac{\mathrm{w}_{2}}{\mathrm{MSE}(\text { yaw rate })}+\frac{\mathrm{w}_{3}}{\mathrm{MSE}(\text { sideslip })}
$$

where $\mathrm{w}_{1}, \mathrm{w}_{2}$, and $\mathrm{w}_{3}$ are weighting factors. In order to avoid unrealistic controller outputs that fluctuate rapidly, a penalty has been added to fitness function values corresponding to simulation results with a high number of oscillations. The genetic algorithm was run for 50 generations, each of which had a population size of 100 chromosomes, a crossover rate of $95 \%$, and a mutation rate of $15 \%$. In addition, an elite selection rate of $2 \%$ was employed to ensure that the fittest chromosomes were retained unaltered from one generation to the next. Using the elite selection technique justifies a relatively high mutation rate, thereby facilitating the thorough exploration of the search space without losing the fittest members of each generation.
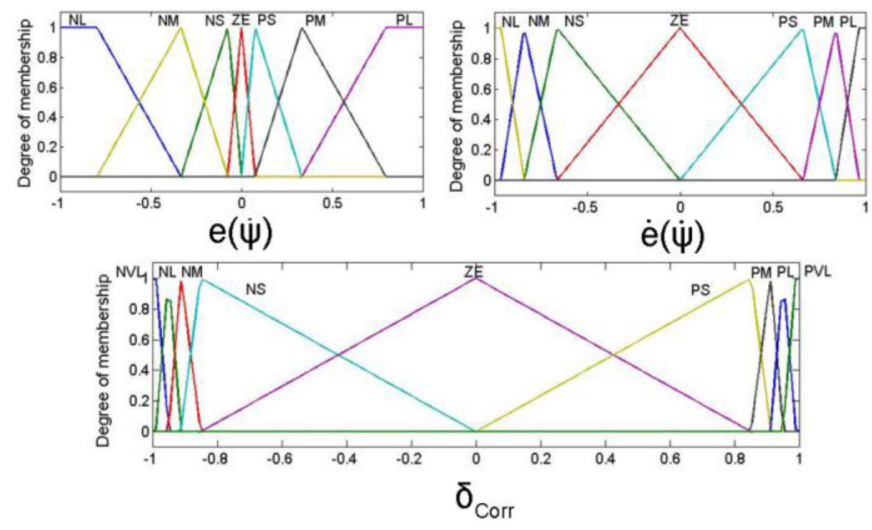

Figure 9. Shapes and distributions of the tuned membership functions for the input and output variables of the fuzzy active steering controller

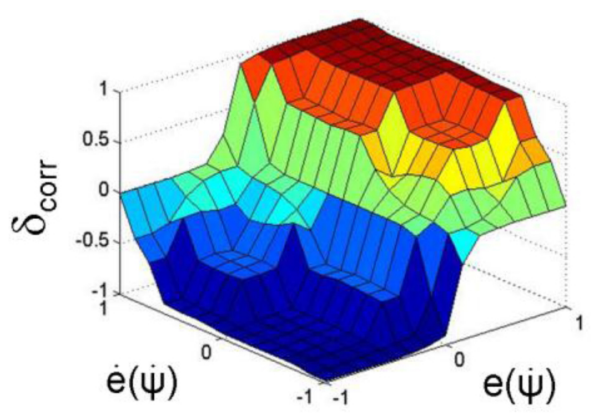

Figure 10. Control surface of the tuned fuzzy active steering controller

The resulting tuned membership functions for the input and output variables are shown in Figure 9. As can be seen, the scaling function has adjusted the shapes and distributions 
of the original (untuned) membership functions (see Figure 5). Figure 9 also provides some insight into the relative importance of each error metric on the stability of the vehicle. For instance, the new arrangement of the membership functions corresponding to the yaw rate error indicates that the controller does not tolerate even small amounts of yaw rate error. The opposite effect can be seen in the membership functions for the rate of change of the yaw rate error: the scaling function has essentially reduced the relative severity of having small errors in this performance metric.

Comparing Figure 10, which illustrates the control surface of the tuned fuzzy active steering controller, with Figure 4 , it is clear that the scaling function has adjusted the membership functions of the output variable of the fuzzy controller such that the control surface extends to the limits of the universe of discourse.

\section{EVALUATION OF THE GENETIC- FUZZY ACTIVE STEERING CONTROLLER}

To confirm the performance of the tuned fuzzy active steering controller, the AUTO21EV is first driven through a double-lane-change maneuver using a driver model [16] with an initial speed of $70 \mathrm{~km} / \mathrm{h}$, both with and without the fuzzy active steering controller. Note that this driver model is considered to represent a professional driver, not an inexperienced average driver. As can be seen in Figure 11, although the driver model is able to steer the vehicle through the maneuver without an active steering controller, the driver required a substantial amount of effort and even countersteered at some points in order to control the vehicle.

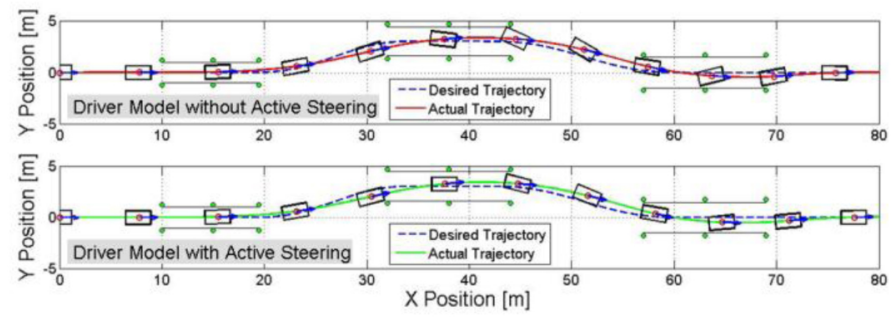

Figure 11. Desired and actual vehicle trajectories when driving through the double-lane-change maneuver using the driver model only (top) and using the driver model with the tuned fuzzy active steering controller (bottom)

On the other hand, the driver was able to negotiate the same maneuver much more easily and smoothly when the fuzzy active steering controller was enabled. This fact is confirmed in Figure 12-a, which illustrates the steering wheel input with respect to time. In order to drive the AUTO21EV through the double-lane-change maneuver, the driver model used the steering wheel more often and more aggressively, and at some points applied up to 300 degrees of steering wheel angle. In contrast, when the fuzzy active steering controller was enabled, the maximum amount of steering wheel angle that the driver applied was about 150 degrees, and at the same time, the rate of change of the steering wheel angle was lower, which indicates an easier and more comfortable drive. Figure 12-b is a handling performance plot that characterizes the relationship between the vehicle yaw rate and the steering wheel input. The closer this plot is to a straight narrow line, the more the vehicle behaves like its reference linear bicycle model, which indicates better steering performance.
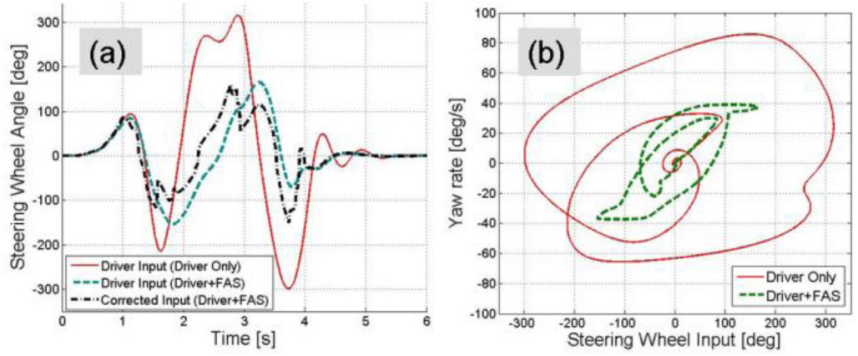

Figure 12. (a) Driver's steering wheel input when driving through the double-lane-change maneuver with (dashed line) and without (solid line) the fuzzy active steering controller, and the equivalent corrected steering wheel angle resulting from the intervention of the fuzzy active steering controller (dash-dotted line); (b) steering wheel input with respect to vehicle yaw rate (handling performance measure)

Figure 13 illustrates the vehicle yaw rate and sideslip angle for this maneuver. Both the vehicle yaw rate and the sideslip angle were smaller when the fuzzy active steering controller was enabled. In addition, the fuzzy active steering controller was able to correct the driver's steering input such that the vehicle performed closer to the desired vehicle behavior represented by the reference bicycle model. Note that driving through this double-lane-change maneuver with an initial speed of $70 \mathrm{~km} / \mathrm{h}$ is considered to be a very aggressive test. Although the fuzzy active steering controller was not able to exactly match the actual vehicle yaw rate with the desired one at this speed, it was confirmed that the controller is capable of this performance at lower speeds.

The second maneuver that is used to test the performance of the fuzzy active steering controller is braking in a turn. This maneuver represents one of the most critical situations encountered in everyday driving, and the reaction of the vehicle to this maneuver reveals the compromise between maneuverability, stability, and braking performance [17]. In this test, the vehicle is driven at a constant speed of $70 \mathrm{~km} / \mathrm{h}$ in a curve with a radius of $60 \mathrm{~m}$. Once the vehicle has entered the curve and has reached a steady-state yaw rate, the driver applies the brakes and slows the vehicle to $20 \mathrm{~km} / \mathrm{h}$. Figure 14 compares the vehicle trajectory observed when the driver model performs the maneuver on its own to the trajectory observed when the driver is assisted by the fuzzy active steering controller. As can be seen, the driver model is unable to control the vehicle without the assistance of the controller: 
the vehicle turns into the curve and leaves the prescribed road. However, as the vehicle slows down, the driver regains control and drives back to the road.

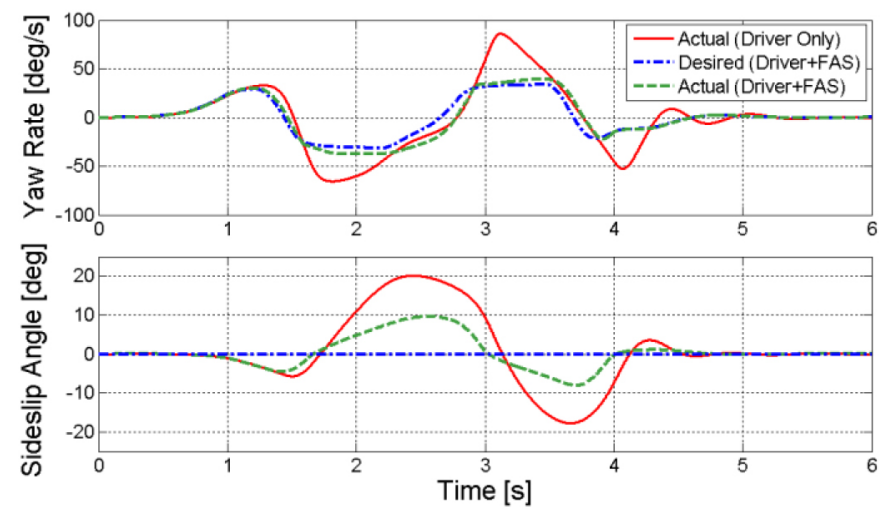

Figure 13. Desired and actual vehicle yaw rates (top) and sideslip angles (bottom) when driving through the double-lane-change maneuver using the driver model with and without the fuzzy active steering controller
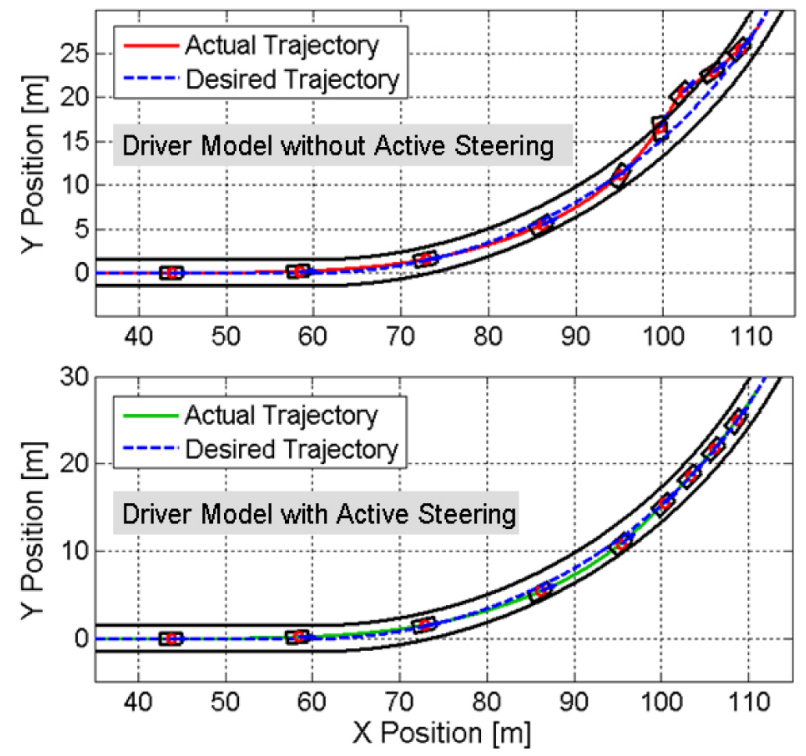

Figure 14. Desired and actual vehicle trajectories when braking in a turn using the driver model only (top) and using the driver model with the fuzzy active steering controller (bottom)

The performance of the fuzzy active steering controller becomes clearer when looking at Figure 15, which compares the vehicle yaw rate and sideslip angle for both cases. When the fuzzy active steering controller is enabled, the driver is able to control the vehicle while braking in the curve, and the vehicle behaves almost like the desired reference bicycle model. It is also interesting to compare the amount of steering wheel input that the driver needs to apply in each case. As shown in Figure 16, even though the unassisted driver model applied the maximum steering wheel input of 700 degrees in a counter-steering action, it could not prevent the vehicle from leaving the road. In contrast, the driver was able to complete the maneuver with a maximum steering wheel input of only 50 degrees and without counter-steering when the fuzzy active steering controller was enabled.

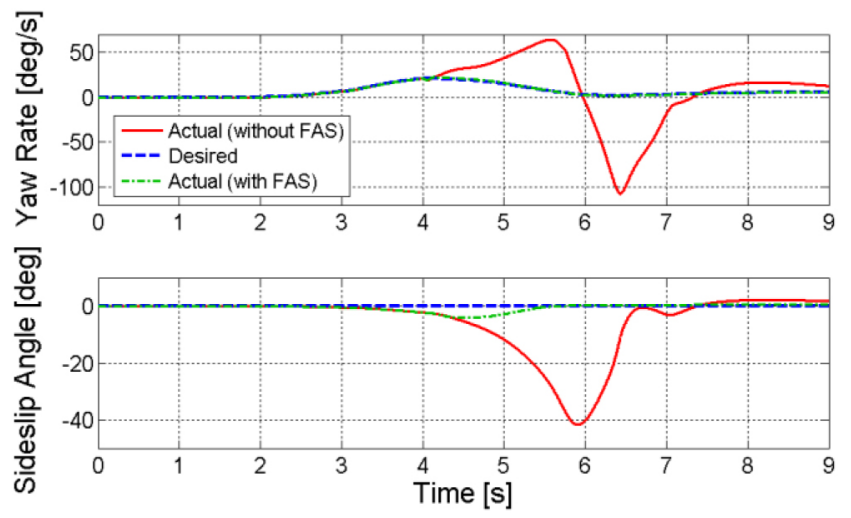

Figure 15. Desired and actual vehicle yaw rates (top) and sideslip angles (bottom) when braking in a turn using the driver model with and without the fuzzy active steering controller
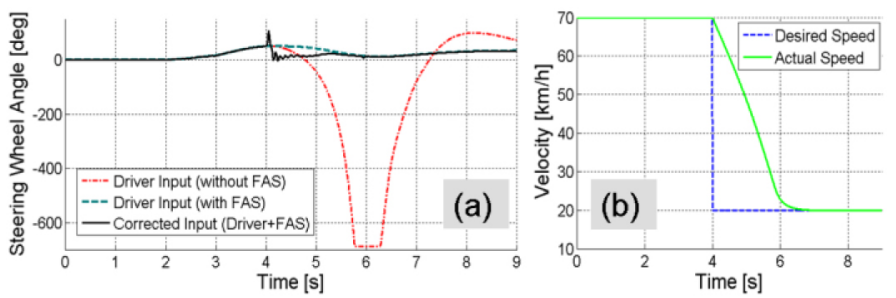

Figure 16. (a) Driver's steering wheel input when braking in a turn with (dashed line) and without (dashdotted line) the fuzzy active steering controller, and the equivalent corrected steering wheel angle resulting from the intervention of the fuzzy active steering controller (solid line); (b) vehicle speed when braking in a turn

A fuzzy active steering system can also be used to correct the side-pushing effect of the vehicle when driving on a $\mu$ split road. As such, we next perform an acceleration test on a $\mu$-split road, where the driver holds the steering wheel fixed and accelerates the vehicle from an initial speed of $10 \mathrm{~km} / \mathrm{h}$ by pressing the accelerator pedal fully, on a road with an ice patch on its left side between $15 \mathrm{~m}$ and $25 \mathrm{~m}$ from the initial vehicle position. Figure 17 illustrates the vehicle trajectory observed during this maneuver and compares it to the case in which the fuzzy active steering controller was disabled. Clearly, the fuzzy active steering controller is capable of correcting the side-pushing effect of the vehicle entirely, without any need for driver compensation. From the vehicle yaw rates and sideslip angles shown in Figure 18, it is apparent that the controller was able to limit and, later, diminish the yaw rate and sideslip angle of the vehicle while driving over the ice patch. The fuzzy active steering 
controller applied an equivalent maximum steering wheel angle of about 50 degrees (Figure 18-b). In short, this maneuver confirms that the cooperation between the fuzzy slip controller that was developed previously [20] and the fuzzy active steering controller developed here has allowed the driver to accelerate the AUTO21EV on a $\mu$-split road with the maximum possible traction forces on all four wheels, no spin-out effects on the wheels, and no side-pushing effect on the vehicle.

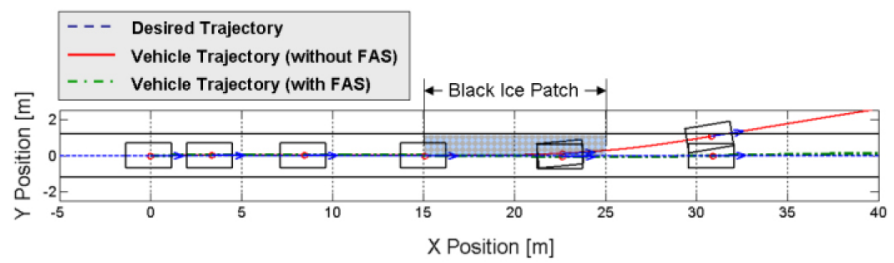

Figure 17. Vehicle trajectories when accelerating on a $\mu$ split road with and without the fuzzy active steering controller
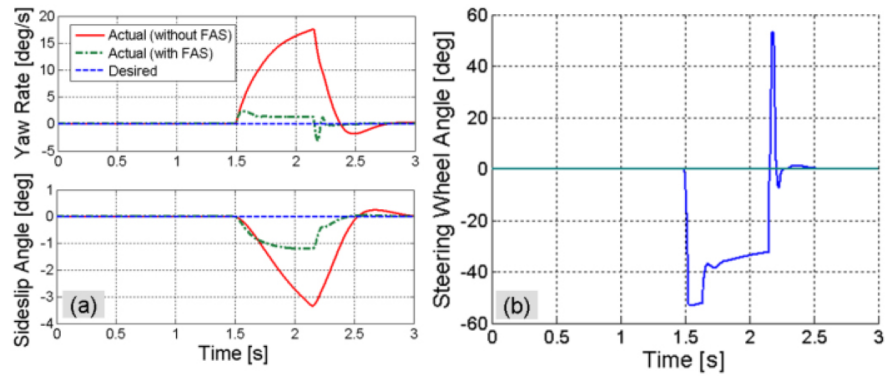

Figure 18. (a) Desired and actual vehicle yaw rates (top) and sideslip angles (bottom) when accelerating on a $\mu$ split road; (b) equivalent steering wheel angle applied by the fuzzy active steering controller in order to correct the side-pushing effect of the vehicle

Finally, we conduct a braking test on a $\mu$-split road, where the driver holds the steering wheel fixed and intends to stop the vehicle in an emergency braking situation from $80 \mathrm{~km} / \mathrm{h}$ on a road that has an ice patch on its left side between $15 \mathrm{~m}$ and $25 \mathrm{~m}$ from the initial position of the vehicle. Figure 19 shows the vehicle trajectory during this maneuver and compares it to the case where the fuzzy active steering controller is disabled. This comparison confirms that the fuzzy active steering controller is able to correct the sidepushing effect of the vehicle while braking on a $\mu$-split road, and can prevent a dangerous instability situation. From the vehicle yaw rates and sideslip angles shown in Figure 20, it is clear that the fuzzy active steering controller was able to limit and, later, diminish the yaw rate and sideslip angle of the vehicle while driving over the ice patch. The fuzzy active steering controller applied an equivalent maximum steering wheel angle of about 50 degrees to counter-steer the vehicle and compensate for the side-pushing effect (Figure 20-b).

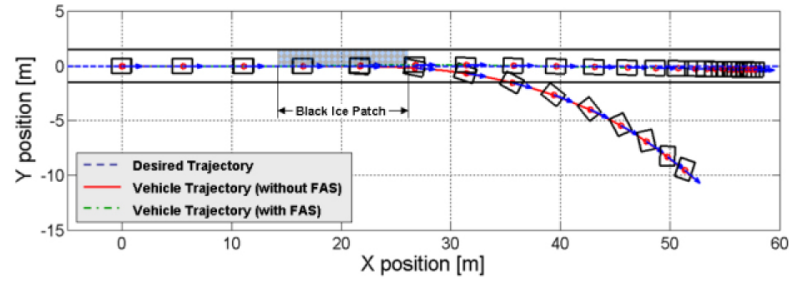

Figure 19. Vehicle trajectories when braking on a $\mu$-split road with and without the fuzzy active steering controller
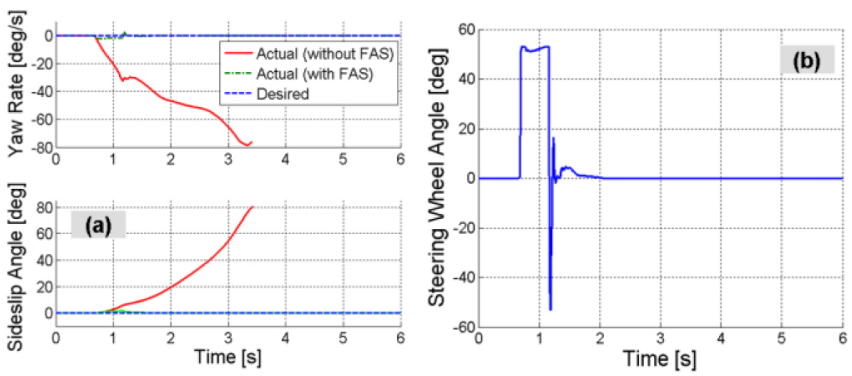

Figure 20. (a) Desired and actual vehicle yaw rates (top) and sideslip angles (bottom) when braking on a $\mu$-split road; (b) equivalent steering wheel angle applied by the fuzzy active steering controller in order to correct the side-pushing effect of the vehicle

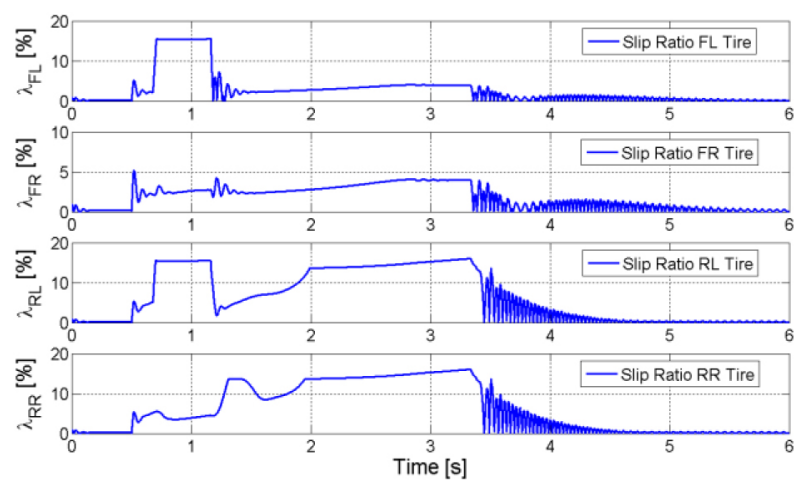

Figure 21. Tire slip ratios when braking on a $\mu$-split road with the fuzzy active steering controller

As shown in Figure 21, the slip controllers on the left side of the vehicle have limited the tire slips when driving over the ice patch, which occurs between 0.7 and 1.2 seconds of the simulation. Furthermore, the slip controllers on the rear wheels have been activated to avoid lock-up, which would have otherwise occurred due to the weight shift to the front axle and the high available braking torques, starting at around 2 seconds; Figure 22, which illustrates the motor torques of all four wheels, confirms this result. It is interesting to note the automatic braking force distribution among the wheels. While the braking torques on the front wheels reach the maximum motor torque of $700 \mathrm{Nm}$ at about 2.8 seconds, the maximum braking torques at the rear wheels are limited to 
$520 \mathrm{Nm}$ by the slip controllers. Furthermore, the braking torques on the front wheels are limited by the driver request at about 3.35 seconds, and on the rear wheels at about 3.42 seconds, which gradually bring the vehicle to a stop at 5 seconds.

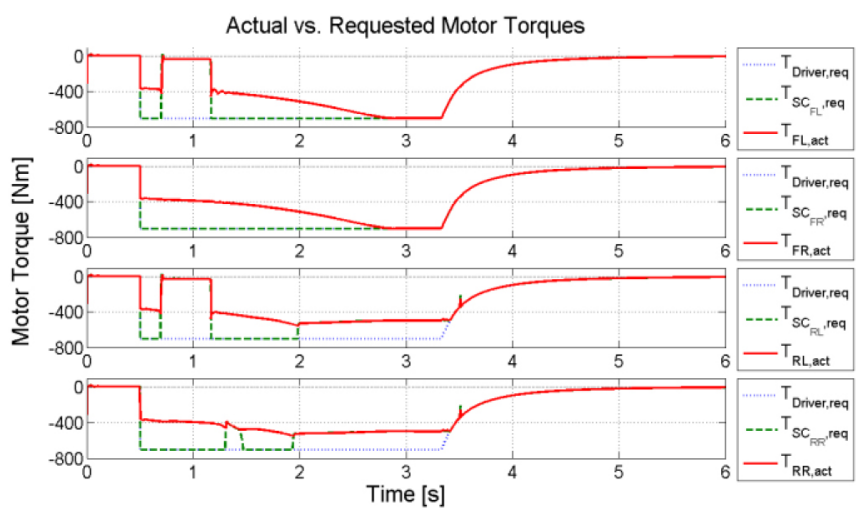

Figure 22. Motor torques when braking on a $\mu$-split road with the fuzzy active steering controller

\section{EVALUATION USING A DRIVING SIMULATOR}

A hardware-in-the-loop (HIL) and operator-in-the-loop (OIL) driving simulator has been used to further evaluate the effectiveness of the proposed controller. HIL simulation refers to the replacement of one or more components of a software model with the analogous hardware component, which communicates with the remaining elements of the software model throughout the simulation. An OIL simulation is similar, except that a human user provides some of the system inputs and observes or otherwise senses some of the system outputs during the simulation. Among the benefits of HIL simulation is its ability to replace preliminary field tests with safer, faster, and more rigorous automated tests. In addition, extreme or unusual situations can be simulated on demand and replicated precisely, enabling the repeated simulation of cold-start engine tests in the summer, for example [18]. It is for these reasons that HIL testing has continued to increase in popularity.

The driving simulator used in this work is shown in Figure 23, and involves the synchronized operation of several pieces of specialized hardware. The vehicle model, in-wheel motor models, and advanced slip controllers have been implemented on a quad-core Peripheral Component Interconnect (PCI) Extensions for Instrumentation (PXI) system from National Instruments, which uses the Laboratory Virtual Instrument Engineering Workbench (LabVIEW) realtime operating system to maintain precise timing during the simulation. Due to the amount of computation involved, a vehicle model with a torque driver applied to each wheel has been implemented on one Central Processing Unit (CPU) core of the quad-core PXI system, and the four in-wheel motor models are executed on a separate core. The advanced slip controllers [20] are run on the third CPU core, receiving sensor signals from the vehicle model and broadcasting control signals at regular intervals. A Windows-based laptop running LabVIEW communicates with the PXI system over Ethernet throughout the simulation. In addition to generating graphical feedback, the laptop must receive universal serial bus (USB) interrupts generated by the steering wheel and pedals, and send the relevant information to the PXI system for use in the simulation. The fuzzy active steering controller has been implemented on an Electronic Control Module (ECM) from MotoTron, which has capabilities similar to the hardware that would be used for this purpose in a production vehicle. The ECM communicates with the PXI system over a Controller Area Network (CAN) bus, which is the standard in vehicular communication networks [19]. The ECM implementation of the fuzzy active steering controller is evaluated using the same maneuvers as before.

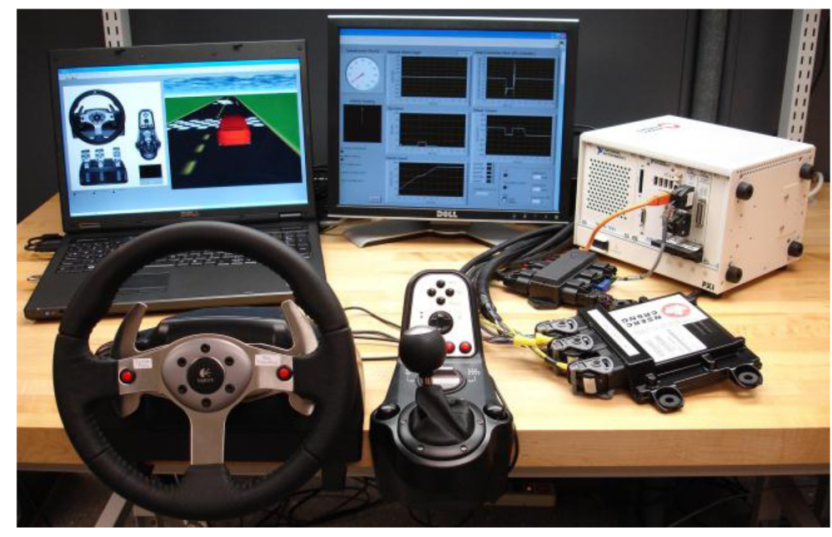

Figure 23. Hardware- and operator-in-the-loop driving simulator equipment

Four maneuvers are used to test the performance and effectiveness of the genetic-fuzzy active steering controller. The first maneuver is the ISO 3888 double-lane-change maneuver described earlier. In order to make this maneuver feasible for a non-professional human driver, however, it was performed with an initial speed of $60 \mathrm{~km} / \mathrm{h}$. Even with this modification, the maneuver was found to be quite challenging without the active steering controller, requiring rapid handover-hand steering and considerable concentration. With the controller enabled, however, the maneuver was found to require substantially less effort. As shown in Figure 24-a, the maneuver was successfully completed both with and without the active steering controller. When the controller was activated, however, the steering effort required by the driver was noticeably reduced, as shown in Figure 24-b. Note, in particular, the steering effort required to recover from the second lane change without the controller. Figure 25-a shows the same driving performance plot as that shown in Figure $\underline{12-b}$, once again indicating that the steering performance of the vehicle is improved by the active steering controller. Finally, the desired and actual yaw rates with and without the 

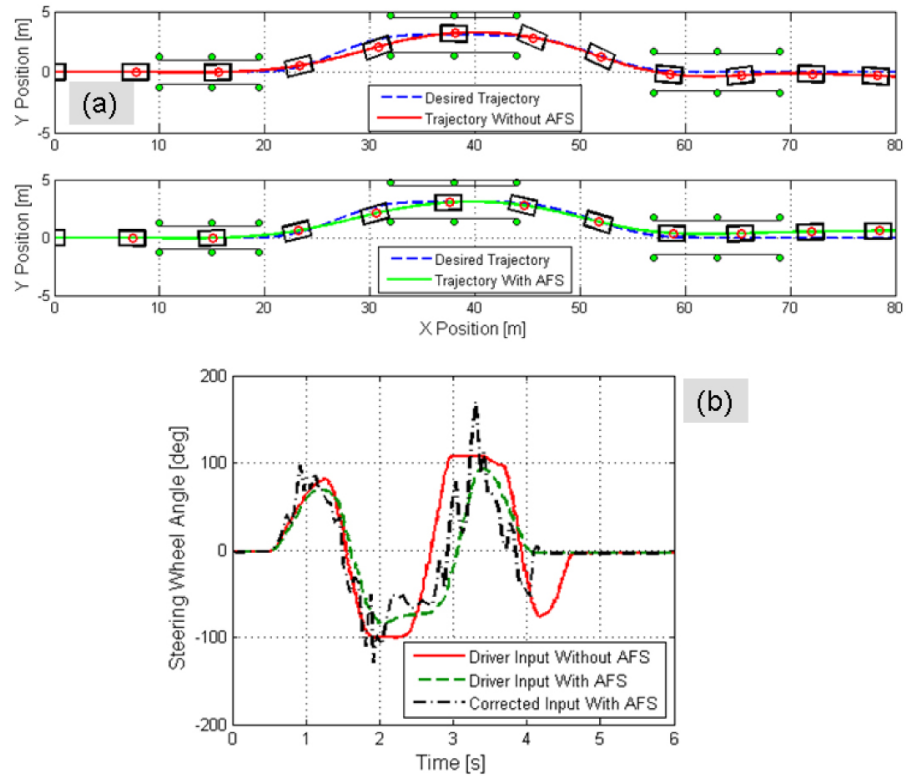

(b)

\section{Figure 24. (a) Vehicle trajectory and (b) steering wheel angle during the double-lane-change maneuver using the driving simulator}

controller are shown in Figure 25-b, which closely match the previous results shown in Figure 13.

The second maneuver used to evaluate the performance of the fuzzy active steering controller is braking in a turn. As before, the vehicle enters a curve with a radius of $60 \mathrm{~m}$ traveling at $70 \mathrm{~km} / \mathrm{h}$. Once a steady-state yaw rate has been reached, the speed is decreased to $20 \mathrm{~km} / \mathrm{h}$ (Figure 26-a). Shown in Figures 26-b and 26-c are the trajectories of the vehicle without and with the aid of the fuzzy active steering controller, respectively. Once again, substantial amounts of driver effort and concentration were required to complete the maneuver without the controller, and even after many attempts, it was not possible to keep the front-left wheel from leaving the road. Only a small amount of driver intervention was required when the controller was active, and it was much easier to maintain the desired trajectory.

Figure 27-a shows the desired and actual vehicle yaw rates with and without the controller. Clearly, the behavior of the vehicle is very close to that of the desired reference bicycle model when using the controller. The steering wheel angles shown in Figure 27-b illustrate the amount of driver effort required with and without the controller. Note, in particular, the large amount of counter-steering required without the controller.

Accelerating and braking on a $\mu$-split road are used next to test the effectiveness the genetic-fuzzy active steering controller. The trajectory of the vehicle when accelerating on a $\mu$-split road is shown in Figure 28-a. Once again, the fuzzy active steering controller is capable of maintaining the desired trajectory when the left tires are on ice.

The data shown in Figures 28-b and 28-c also agree with the results found previously, as illustrated in Figures 18-a and $\underline{18-b}$. Finally, we repeat the straight-line braking maneuver on a $\mu$-split road with the fuzzy active steering controller. Recall that, without a corrective steering input, the vehicle yaws considerably and leaves the road. The trajectory when assisted by the fuzzy active steering controller is nearly identical to the desired trajectory (Figure 29). The tire slip ratios and motor torques shown in Figures 30 and $\underline{31}$ are in very close agreement with the previous results shown in Figures 21 and 22 , respectively.

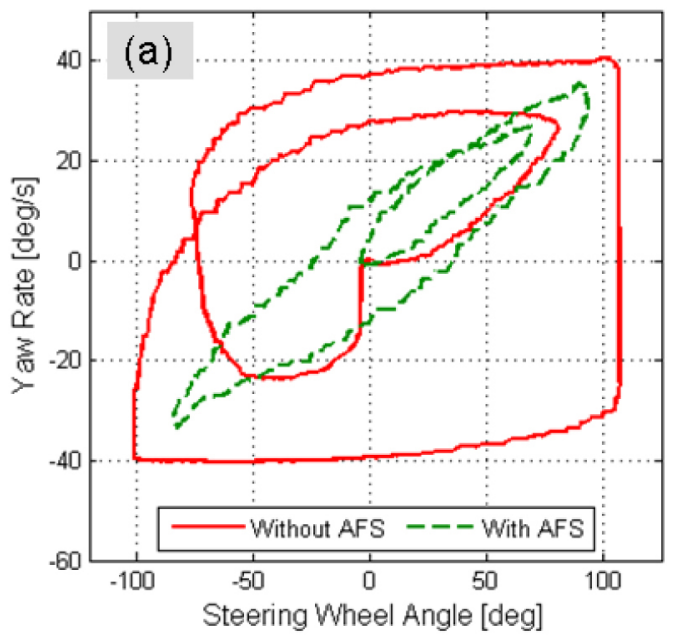

Figure 25. (a) Vehicle yaw rate with respect to the driver's steering wheel angle and (b) vehicle yaw rate with respect to time during the double-lane-change maneuver using the driving simulator 


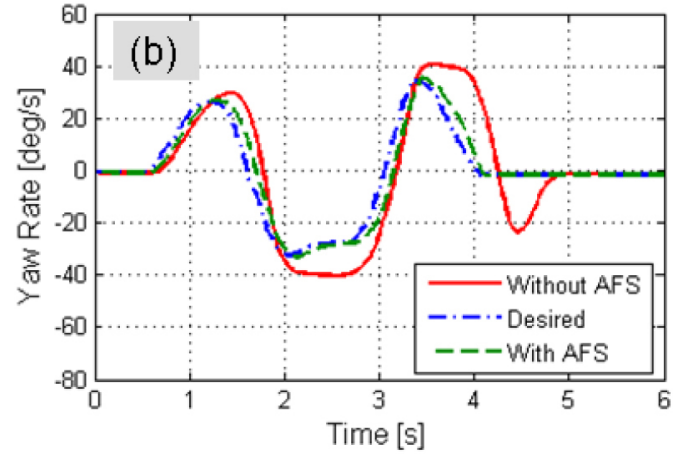

Figure 25 (cont.). (a) Vehicle yaw rate with respect to the driver's steering wheel angle and (b) vehicle yaw rate with respect to time during the double-lane-change maneuver using the driving simulator
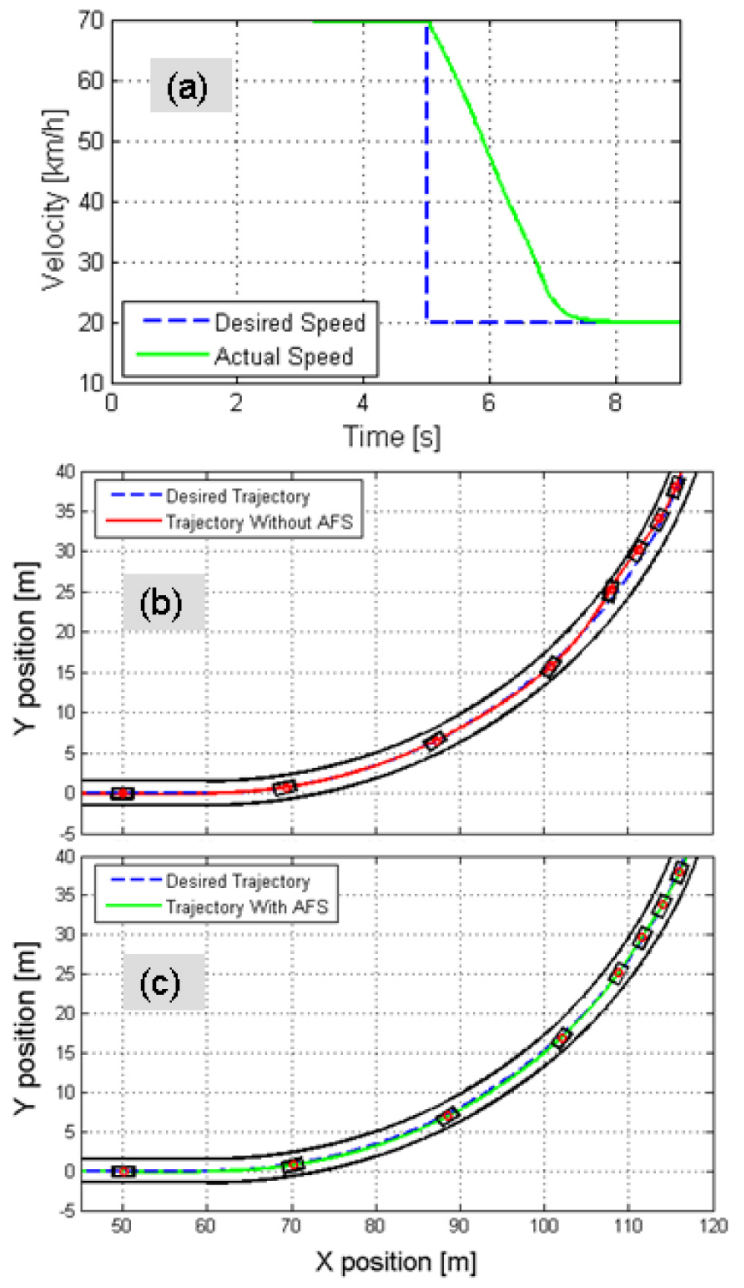

Figure 26. (a) Vehicle speed, (b) trajectory without the fuzzy active steering controller, and (c) trajectory with the fuzzy active steering controller when braking in a turn using the driving simulator
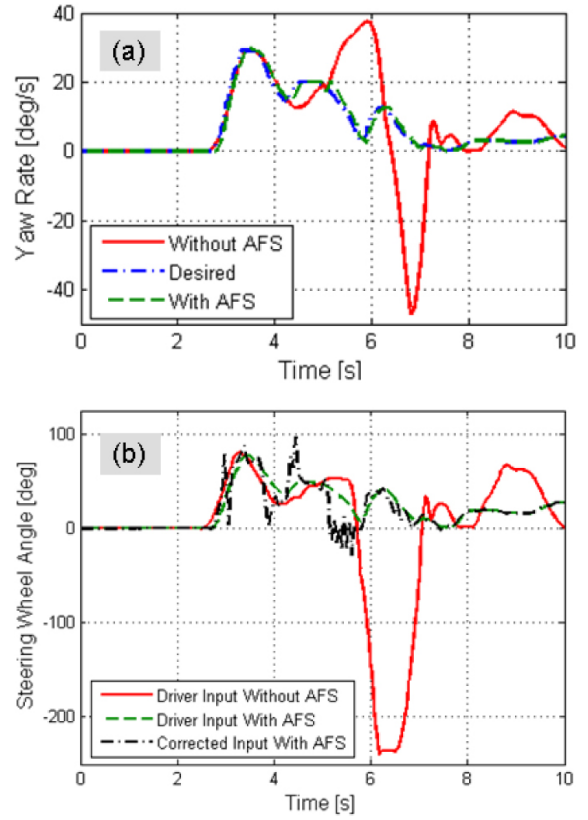

Figure 27. (a) Vehicle yaw rate and (b) driver's steering wheel angle when braking in a turn using the driving simulator
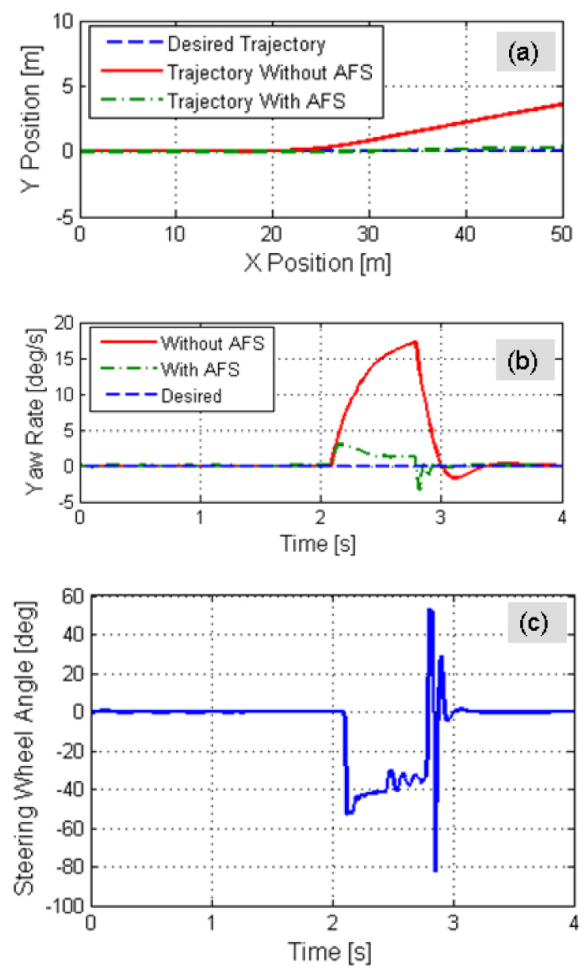

Figure 28. (a) Vehicle trajectory, (b) yaw rate, and (c) equivalent steering wheel angle applied by the fuzzy active steering controller when accelerating on a $\mu$-split road using the driving simulator 


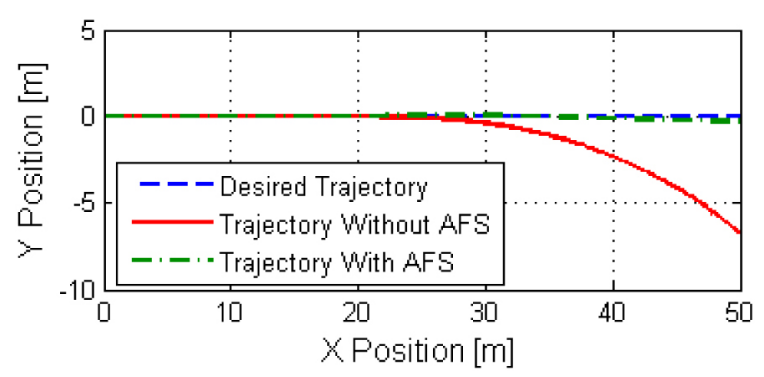

Figure 29. Vehicle trajectory when braking on a $\mu$-split road using the driving simulator

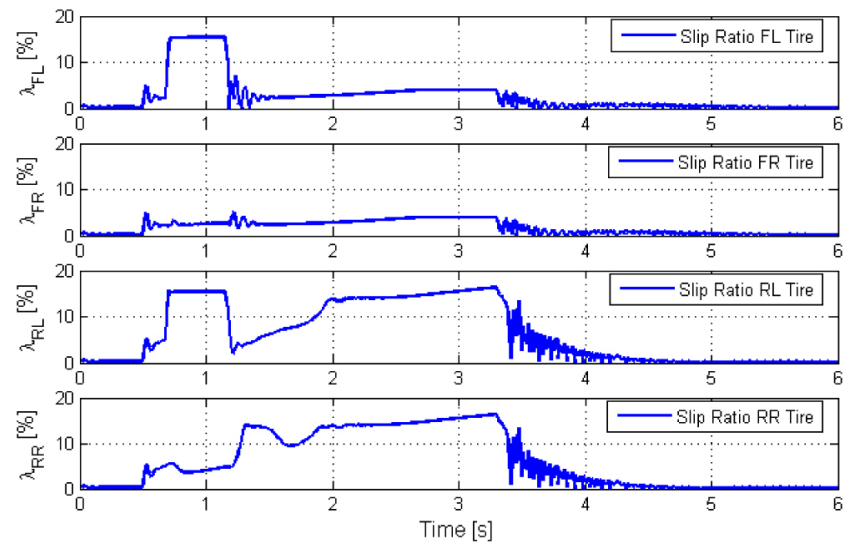

Figure 30. Tire slip ratios when braking on a $\mu$-split road using the driving simulator with the fuzzy active steering controller

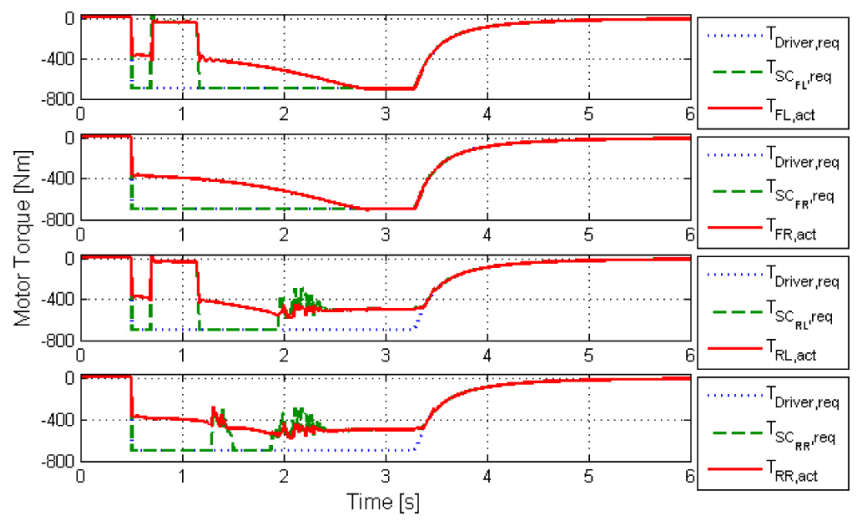

Figure 31. Motor torques when braking on a $\mu$-split road using the driving simulator with the fuzzy active steering controller

\section{CONCLUSIONS}

An electric vehicle model with four direct-drive in-wheel motors and an active steering system has been developed using ADAMS/View and MapleSim, a multi-domain modeling package that can generate and export computationally efficient simulation code through the use of linear graph theory and symbolic computing. Electric vehicles are excellent platforms on which to apply advanced motion control techniques, since the motor speeds and torques can be generated and controlled quickly, precisely, and independently for each wheel. The MapleSim model has been used to design and develop a novel genetic-fuzzy active steering controller. The objective of the controller is to determine the corrective steering angle required to stabilize the vehicle by minimizing the vehicle yaw rate and sideslip angle errors. A hybrid genetic-fuzzy approach is used to tune the controller, optimizing the shapes and distributions of its membership functions. By combining a genetic algorithm with a fuzzy control system, each soft computing paradigm can compensate for the weaknesses of the other, and a more powerful hybrid technique can emerge. A multi-criteria genetic algorithm is used to tune the membership functions of the input and output variables of the fuzzy active steering controller such that the vehicle trajectory, yaw rate, and sideslip angle errors are minimized. The performance of the final genetic-fuzzy active steering controller is examined during a severe double-lane-change maneuver, when braking in a turn, and while accelerating and braking on a $\mu$-split road. The preliminary results indicate that the proposed genetic-fuzzy active steering controller has the ability to improve the performance of the vehicle handling and stability considerably. As the final evaluation step, the genetic-fuzzy active steering controller is implemented on an external electronic control module communicating with a hardwareand operator-in-the-loop driving simulator. The results from the driving simulator confirm the excellent performance of the genetic-fuzzy active steering controller in all four driving maneuvers.

\section{ACKNOWLEDGMENTS}

Funding for this work was provided by the Natural Sciences and Engineering Research Council of Canada and a grant from AUTO21, a Canadian Network of Centres of Excellence.

\section{REFERENCES}

1. Koehn, P. and Eckrich, M., "Active Steering - The BMW Approach Towards Modern Steering Technology," SAE Technical Paper 2004-01-1105, 2004, doi:10.4271/2004-01-1105.

2. Yih, P.: "Steer-by-wire: Implications for vehicle handling and safety", $\mathrm{PhD}$ dissertation, Department of Mechanical Engineering, Stanford University, 2005.

3. Mammar, S. and Koenig, D.: "Vehicle handling improvement by active steering", Vehicle System Dynamics: International Journal of Vehicle Mechanics and Mobility, 38(3), pp. 211-242, 2002.

4. Knoop, M., Leimbach, K., and Shröder, W., "Increased Driving Comfort and Safety by Electronic Active Steering," SAE Active Safety TOPTEC, Sept. 1999.

5. Ackermann, J.: "Active steering for better safety, handling and comfort", Proceedings of Advances in Vehicle Control and Safety, Amiens, pp. 1-10, 1-3 July 1998.

6. Reinelt, W., Klier, W., Reimann, G., Schuster, W. et al., "Active Front Steering (Part 2): Safety and Functionality," SAE Technical Paper 2004-01-1101, 2004, doi:10.4271/2004-01-1101. 
7. Bode, K.H.: Preliminary Suspension Design and Stability Control Strategies for an Electric Vehicle with Four Independently Driven InWheel Motors, Project Thesis, University of Waterloo, 2006.

8. Karray, F.O. and de Silva, C.: Soft Computing and Intelligent Systems Design: Theory, Tools and Applications, Pearson Education Limited, 2004.

9. Manning, W.J. and Crolla, D.A.: "A review of yaw rate and sideslip controllers for passenger vehicles", Transactions of the Institute of Measurement and Control, 29(2), pp. 117-135, 2007.

10. Gordon, T., Howell, M., and Brandao, F.: "Integrated control methodologies for road vehicles", Vehicle System Dynamics: International Journal of Vehicle Mechanics and Mobility, 40(1-3), pp. 157-190, 2003.

11. Andreasson, J., Knobel, C., and Bünte, T.: "On road vehicle motion control - striving towards synergy", Proceedings of the 8th International Symposium on Advanced Vehicle Control, Taipei, AVEC060209, 20-24 August 2006.

12. van Zanten, A., "Bosch ESP Systems: 5 Years of Experience," SAE Technical Paper 2000-01-1633, 2000, doi:10.4271/2000-01-1633.

13. Paine, M.: "Electronic stability control: review of research and regulations", Vehicle Design and Research Pty Limited, Report No. G248, 2 June 2005.

14. Bauer, H.: Driving Safety Systems, 2nd edition, Robert Bosch GmbH, Warrendale, 1999.

15. Cordón, O., Herrera, F., Hoffmann, F., and Magdalena, L.: Genetic Fuzzy Systems: Evolutionary Tuning and Learning of Fuzzy Knowledge Bases, World Scientific Publishing, 2001.

16. Jalali, K., Lambert, S., and McPhee, J., "Development of a PathFollowing and a Speed Control Driver Model for an Electric Vehicle," SAE Int. J. Passeng. Cars - Electron. Electr. Syst. 5(1):100-113, 2012, doi: $10.4271 / 2012-01-0250$.

17. ISO Passenger Cars - Braking in a Turn: Open-loop Test Method, ISO 7975:2006.

18. Krisp, H., Lamberg, K., and Leinfellner, R., "Automated Real-Time Testing of Electronic Control Units," SAE Technical Paper 2007-01-0504, 2007, doi:10.4271/2007-01-0504

19. Bosch Automotive Handbook, 6th edition, Robert Bosch GmbH, Plochingen, 2004.

20. Jalali, K., Uchida, T., McPhee, J., and Lambert, S., "Development of a Fuzzy Slip Control System for Electric Vehicles with In-wheel Motors," SAE Int. J. Alt. Power. 1(1):46-64, 2012, doi:10.4271/2012-01-0248. 